\title{
Manejo de la insuficiencia cardiaca con estimulación hisiana en bloqueo de rama derecha: reporte de caso
}

\author{
Management of heart failure with His bundle pacing in right bundle branch block: case report \\ Jorge Gómez-Flores ${ }^{1}$, Jovana Pérez-Báez ${ }^{1 *}$, Guillermo Muñoz ${ }^{1}$, Gabriela Bustillos-García ${ }^{1}$, Alan García1, \\ Álvaro E. Reyes-Quintero', Rosa M. Ávila-Ocampo², Antonio Arias-Godínez², Manlio Márquez', \\ Luis Colin-Lizalde ${ }^{1}$, Moisés Levinstein-Jacinto ${ }^{1}$, José L. Morales-Velázquez ${ }^{1}$ y Santiago Nava ${ }^{1}$ \\ ${ }^{1}$ Departamento de Electrofisiología; 2 Departamento de Ecocardiografía. Instituto Nacional de Cardiología Ignacio Chávez, Ciudad de México, México
}

\begin{abstract}
Resumen
La terapia de resincronización cardiaca mediante estimulación hisiana ha demostrado ser efectiva en pacientes con bloqueo de rama izquierda del haz de His e insuficiencia cardiaca. Paciente masculino, con 47 años de edad, con insuficiencia cardiaca, fracción de expulsión del $17 \%$ y miocardio dilatada idiopática, electrocardiograma en ritmo sinusal, bloqueo auriculoventricular de $1 .^{\text {er }}$ grado, intervalo PR 400 ms, bloqueo completo de rama derecha del haz de His, bloqueo del fascículo anterior de la rama izquierda del haz de His, duración del QRS 200 ms. Se decidió realizar estimulación selectiva del haz de His. La resincronización cardiaca biventricular convencional en pacientes con presencia de bloqueo completo de la rama derecha del haz de His no está indicada debido a la pobre respuesta al tratamiento. La estimulación hisiana permite reclutar la rama bloqueada y reestablecer la conducción a través de ella, de tal forma que, en ausencia de necrosis, se logre sincronía biventricular. En el caso presentado el reclutamiento de la rama derecha mediante estimulación hisiana se reflejó en el restablecimiento de la sincronía biventricular, medida por rastreo de marcas (speckle tracking) e incremento significativo de la fracción de expulsión del ventrículo izquierdo del 17 al 36.6\%, con un incremento absoluto del $19.6 \%$.
\end{abstract}

Palabras clave: Resincronización. Bloqueo de rama derecha. Estimulación hisiana. Insuficiencia cardiaca. México.

\begin{abstract}
Cardiac resynchronization therapy has proven to be an effective therapy in patients with left bundle branch block and heart failure. Male, 47 years old, heart failure with a left ventricle ejection fraction of 17\%, idiopathic heart failure. ECG with sinus rhythm, $1^{\text {st }}$ degree AV block, PR $400 \mathrm{~ms}$, complete right bundle branch block, anterior hemi-fascicle of the left bundle of His, and QRS duration $200 \mathrm{~ms}$. We decided to perform a selective His bundle pacing. In patients with right bundle branch block the biventricular cardiac resynchronization is not indicated due to low treatment response. His bundle pacing allows recruiting the blocked branch and restoring conduction throughout it, therefore, in the absence of necrosis the biventricular synchrony is achieved. We presented a case of His bundle pacing with recruitment of the right bundle branch, which reestablish biventricular synchrony measured by speckle tracking, and with a significant increase of the left ventricle ejection fraction from 17 to $36.6 \%$, with an absolute increase of $19.6 \%$.
\end{abstract}

Key words: Cardiac resynchronization. Right bundle branch block. His bundle pacing. Heart failure. México.

Correspondencia:

*Jorge Gómez-Flores

E-mail: jorge.gomez@cardiologia.org.mx
Disponible en internet: 23-02-2021

Arch Cardiol Mex. 2021;91(1):93-99 www.archivoscardiologia.com

(a) 2020 Instituto Nacional de Cardiología Ignacio Chávez. Publicado por Permanyer. Este es un artículo open access bajo la licencia CC BY-NC-ND (http://creativecommons.org/licenses/by-nc-nd/4.0/).

Fecha de recepción: 17-12-2019

Fecha de aceptación: 05-11-2020 DOI: 10.24875/ACM.19000402 


\section{Introducción}

La terapia de resincronización cardiaca (TRC) consiste en el implante de un dispositivo de estimulación biventricular (puede o no acompañarse de desfibrilación) para pacientes con insuficiencia cardiaca que tiene como objetivo mejorar la sincronía eléctrica auriculoventricular, interventricular e intraventricular, así como el volumen sistólico del ventrículo izquierdo ${ }^{1}$.

Las guías de práctica clínica indican el uso de la TRC para los pacientes con insuficiencia cardiaca (New York Heart Association [NYHA] II-IV), fracción de expulsión del ventrículo izquierdo (FEVI) $<35 \%$, QRS $>150 \mathrm{~ms}$ y morfología de bloqueo de rama izquierda del haz de His (BRIHH) (indicación de clase I, nivel de evidencia A)². No existen estudios concluyentes que demuestren el beneficio de la estimulación biventricular en presencia de bloqueo de rama derecha del haz de His $(\mathrm{BRDHH})^{3}$. Sin embargo, las guías actuales de TRC proveen como recomendación clase lla: para pacientes con FEVI $\leq 35 \%$, ritmo sinusal, patrón de bloqueo distinto a BRIHH, QRS $\geq 150$ ms y NYHA clase III; y llb para pacientes con patrón de bloqueo distinto a BRIHH y QRS de 120 a $150 \mathrm{~ms}^{4}$.

La estimulación selectiva del haz de His permite el reclutamiento del sistema His-Purkinje nativo, en aquellos pacientes con trastorno de conducción interventricular (BRIHH, BRDHH) y bloqueo atrioventricular (AV) supra-e infra-hisiano; de tal forma que mejora la sincronía interventricular y la $\mathrm{FEVI}^{5}$.

La estimulación selectiva del haz de His se puede realizar con éxito de forma simultánea con estimulación del ventrículo izquierdo con electrodo en el seno coronario (His-optimized CRT o "HOT CRT") ${ }^{6}$. Existe la posibilidad técnica de colocar el electrodo de estimulación del haz de His en el puerto de conexión del ventrículo derecho del generador de TRC reduciendo la amplitud del QRS y generando resincronización adecuada. Actualmente el método más usado es colocar el electrodo Select Secure (Medtronic Inc, Minneapolis, EUA) conectado al puerto del ventrículo derecho de un marcapaso convencional, ya sea unicameral con presencia de fibrilación auricular o bicameral ${ }^{7}$.

Se presenta un caso de estimulación del haz de His como método de resincronización.

\section{Caso clínico}

Hombre de 47 años, con diagnóstico de miocardiopatía en fase dilatada, idiopática, insuficiencia cardiaca en clase funcional III de la NYHA, trastorno de conducción intraventricular, interventricular y auriculoventricular por la presencia de BRDHH, bloqueo fascicular anterior (BFA) y bloqueo AV de 1 er $^{\text {erado. La }}$ insuficiencia cardiaca se agudizó cuatro meses previo al ingreso, a pesar de tratamiento médico máximo, con evolución caracterizada por edema de miembros inferiores, disnea de moderados a pequeños esfuerzos, disnea paroxística nocturna y ortopnea (clase funcional NYHA IV); mareo, visión borrosa y síncope en tres ocasiones con recuperación ad integrum; por lo cual recibió atención hospitalaria documentando en ese momento bloqueo AV completo transitorio que alternaba con ritmo sinusal, BRDHH y BFA de base. En el ecocardiograma transtorácico basal se informó FEVI del $17 \%$, aurícula y ventrículo izquierdo dilatados e hipocinesia global del ventrículo izquierdo. En el estudio de perfusión miocárdica por medicina nuclear realizado con tecnecio 99 se observó isquemia grave inferoapical y septobasal e isquemia moderada en segmento medio de la pared anterior. Se realizó angiografía coronaria en la cual se demostró ausencia de lesiones coronarias.

El electrocardiograma (ECG) basal (Fig. 1) en ritmo sinusal con bloqueo AV de 1 er $^{\text {er grado con intervalo } P R}$ de $400 \mathrm{~ms}$, frecuencia cardiaca (FC) $66 \mathrm{lpm}$, eje eléctrico del QRS $-60^{\circ}$, QRS $200 \mathrm{~ms}$, BRDHH y BFA.

Por resonancia magnética nuclear simple y contrastada se observaron diámetros de las cavidades izquierdas dilatadas, disfunción sistólica biventricular grave, FEVI 19\%, la fracción de eyección del ventrículo derecho al $15 \%$ y zonas difusas de reforzamiento tardío con patrón sugestivo de miocarditis no activa.

Durante la hospitalización presentó un episodio de taquicardia ventricular monomórfica sostenida, sin compromiso hemodinámico, que se manejó con amiodarona. Debido a los datos de insuficiencia cardiaca agudos, la clase funcional III de la NYHA, el QRS de $180 \mathrm{~ms}$, la presencia de bloqueo AV de $1 .^{\text {er }}$ grado y BRDHH asociado a BFA, se decidió la realización de estimulación selectiva del haz de His más desfibrilador implantable con TRC (TRC-D).

\section{Descripción del procedimiento}

Mediante acceso venoso femoral se colocó catéter cuadripolar en la zona del haz de His, se registró el potencial en el polígrafo y se confirmó bloqueo AV infrahisiano, con una duración del intervalo HV de 147 ms.

Previa asepsia y antisepsia de la región infraclavicular izquierda, se realizó incisión deltopectoral izquierda, disección por planos y marsupialización subcutánea. 


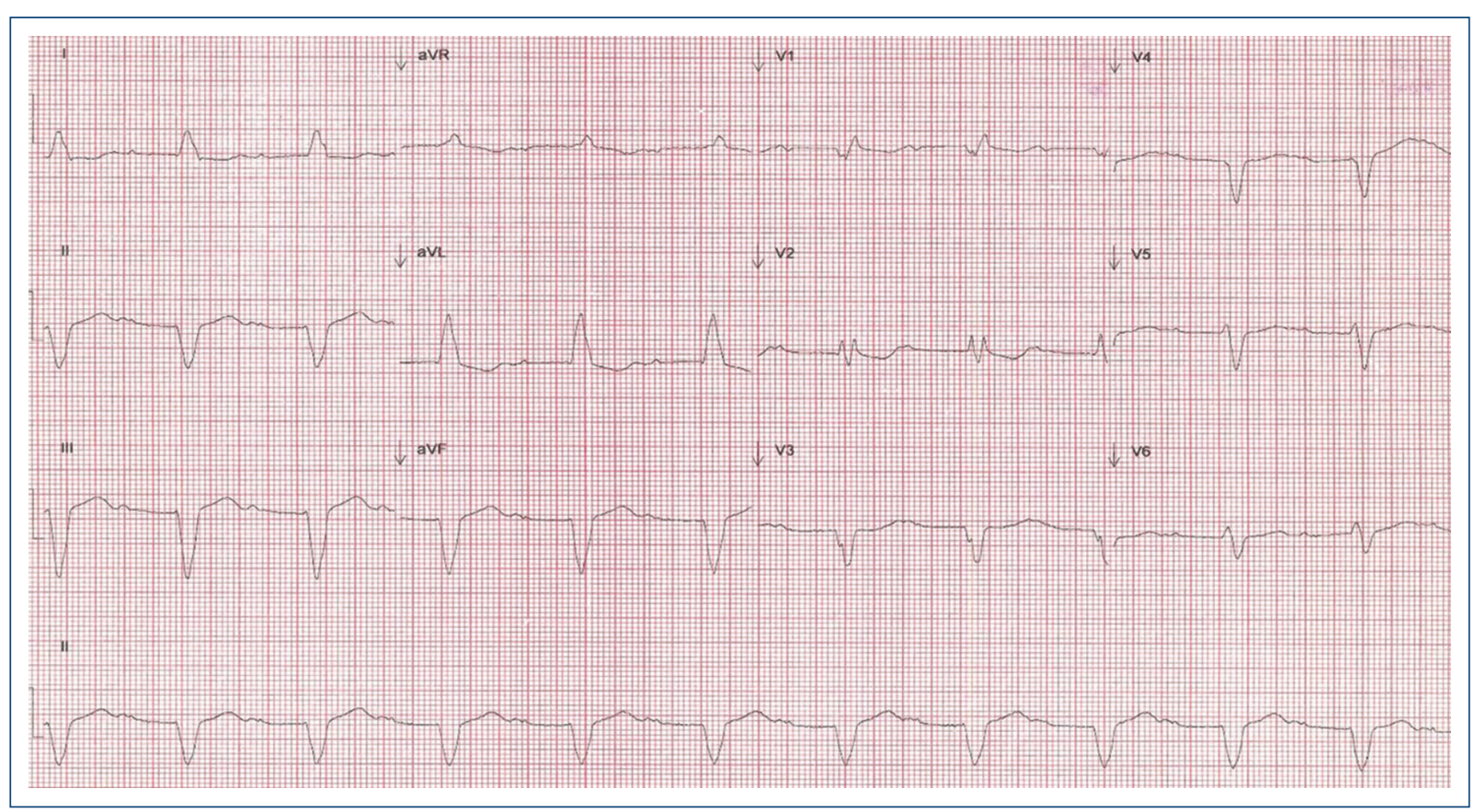

Figura 1. Electrocardiograma previo a la estimulación hisiana. Ritmo sinusal con bloqueo atrioventricular de 1.er grado con intervalo PR de $400 \mathrm{~ms}$, frecuencia cardiaca $66 \mathrm{lpm}$, eje eléctrico del QRS $-60^{\circ}$, ORS $200 \mathrm{~ms}$, bloqueo de rama derecha del haz de His y bloqueo del fascículo anterior.

Se obtuvo acceso venoso mediante punción de la vena subclavia izquierda con aguja de Seldinger, colocando las guías metálicas a la vena cava inferior. Se colocó introductor $6 \mathrm{Fr}$, a través del cual se avanzó la vaina C315 HIS (Medtronic Inc, Minneapolis, EUA) preformada, y a través de ella se avanzó el electrodo Select Secure (Medtronic Inc, Minneapolis, EUA) hacia el haz de His, una vez en el sitio de mejor registro del His con onda $\mathrm{R}$ de $16 \mathrm{mV}$, se estimuló en modo unipolar a $10 \mathrm{mV}$ con ancho de pulso $1 \mathrm{~ms}$, en el ECG de 12 derivaciones se observó disminución de la duración del intervalo QRS a140 ms, se disminuyó progresivamente la salida a $5 \mathrm{~V}$ con incremento de la duración del QRS a $152 \mathrm{~ms}$, hasta obtener captura hisiana con reclutamiento de la rama derecha.

El resto del procedimiento se realizó de manera convencional. El tiempo de fluoroscopia fue de 20 minutos.

Se obtuvo ECG con estimulación: intervalo R-R 929 ms, duración del QRS 160 ms, QT 525 ms. Se dejó programado con los siguientes parámetros: Modo DDD, FC básica 60 ppm, FC máxima 130 ppm, salida auricular $3.5 \mathrm{~V}$, ancho de pulso $0.4 \mathrm{~ms}$, salida en el sitio de estimulación hisiana de $5 \mathrm{~V}$, ancho de pulso $1 \mathrm{~ms}$.

Posterior a 24 horas del procedimiento, se obtuvo ECG con reducción en la duración del QRS (Fig. 2). Se realizó una evaluación ecocardiográfica antes y después de la TRC mediante la técnica de rastreo de puntos (speckle tracking) sin estimulación se observó retraso en la conducción de las paredes anterior y posterior respecto a la pared septal, esto se explica por el BFA (Fig. 3). Posterior a la estimulación hubo mejoría significativa de la asincronía interventricular e intraventricular y la cuantificación de FEVI 3D final con software Syngo en Siemens Accuson S2000 fue del 36.6\% (Fig. 4).

Tras ocho meses de la TRC, el paciente se encuentra asintomático, con mejoría en la calidad de vida y en la clase funcional (NYHA I).

\section{Discusión}

La TRC ha mostrado una mejora en la supervivencia de los pacientes con insuficiencia cardiaca con mejoría de diversos indicadores como la reversión del remodelado del ventrículo izquierdo, incremento de la capacidad de ejercicio, reducción de la tasa de hospitalizaciones por insuficiencia cardiaca y disminución de la tasa de mortalidad ${ }^{8}$. El objetivo de la resincronización es lograr la coordinación del patrón de contracción de las paredes opuestas a aquellas que muestran alteración de la sincronía en el ventrículo izquierdo9.

Sin embargo, en presencia de BRDHH no se ha demostrado beneficio con la TRC; en pacientes con BRD$\mathrm{HH}$ y BFA concomitante, Chandra, et al. ${ }^{10}$ reportaron 


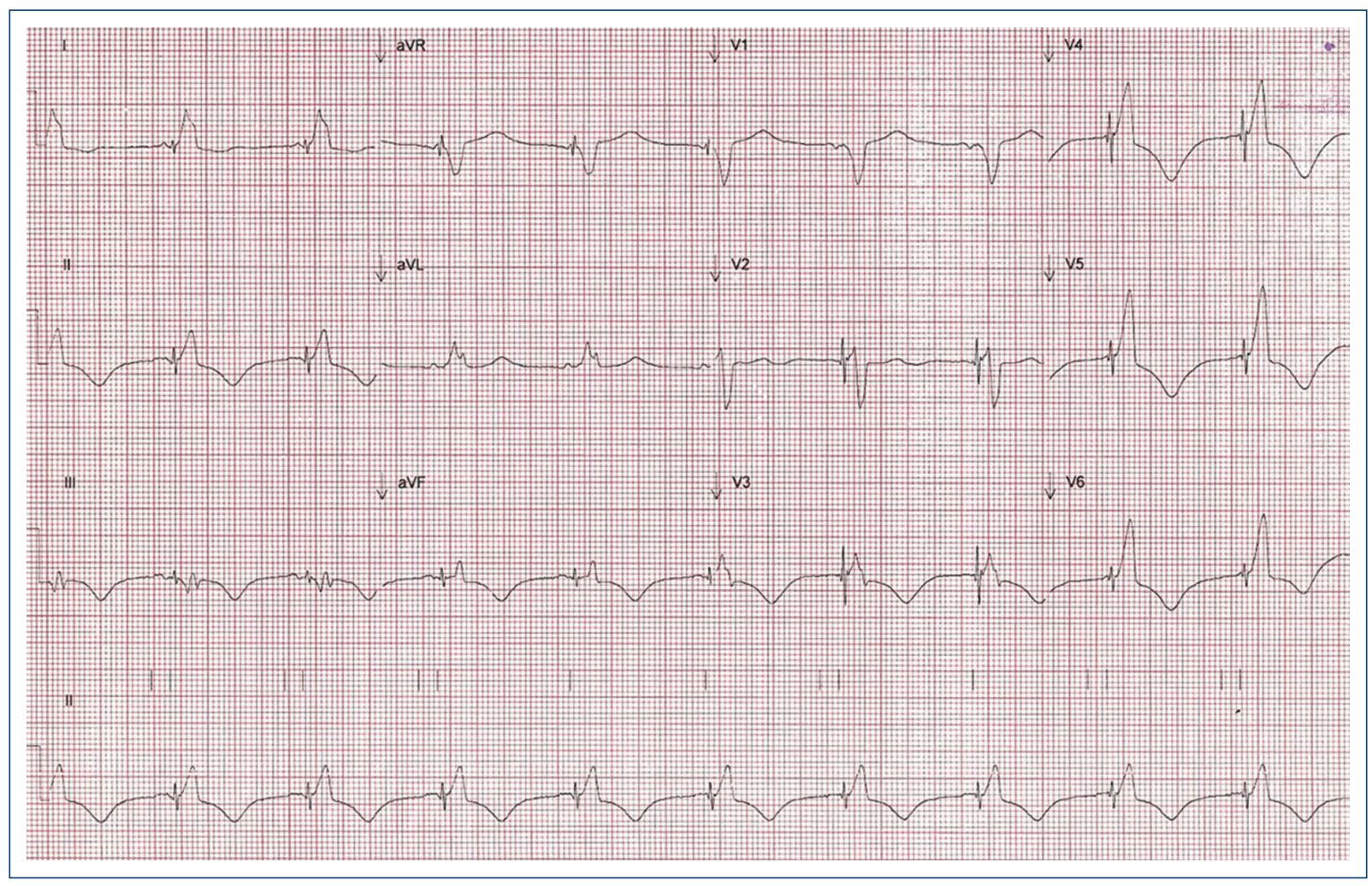

Figura 2. Electrocardiograma posterior a la estimulación hisiana. Intervalo R-R 929 ms, duración del QRS 160 ms, QT $525 \mathrm{~ms}$, bloqueo de rama izquierda del haz de His.

una mejor respuesta a la TRC, definida como un incremento de la $\mathrm{FEVI}>5 \%$ o mejora de la clase funcional NYHA. Otro grupo, en cambio (Varma, et al. $\left.{ }^{11}\right)$, estudió el retraso de la activación inferolateral del ventrículo izquierdo, midiendo la distancia entre el inicio del QRS en el ECG y la activación intrínseca del ventrículo izquierdo en el registro endocavitario, en pacientes con disfunción ventricular izquierda y BRDHH, en comparación a un grupo con $\mathrm{BRIHH}$; encontraron una mejor respuesta a la TRC en los pacientes con BRIHH.

La estimulación hisiana ha sido descrita recientemente como alternativa viable a la estimulación biventricular en pacientes con indicación para resincronización cardiaca ${ }^{12}$.

La estimulación en His puede resultar en la corrección o reclutamiento de la rama derecha con estrechamiento del QRS. Considerando que la activación eléctrica en pacientes con BRDHH muestra un retraso en el ventrículo derecho con mínima o nula alteración del ventrículo izquierdo, la sincronización del retraso de activación del ventrículo derecho y activación normal del ventrículo izquierdo es posible mediante estimulación hisiana. Se puede lograr mediante dos estrategias: 1) reclutamiento de la rama derecha del haz de His y normalización del retraso de activación del ventrículo derecho, y 2) en los casos en los que no se logre estimulación selectiva del haz de His, la fusión entre la estimulación hisiana (con patrón de BRDHH) y la estimulación del ventrículo derecho corrige el retraso de la activación y también es efectiva. Un efecto favorable de la estimulación hisiana es que no genera disincronía del ventrículo izquierdo, ya que la activación ocurre por el sistema His-Purkinje; mientras que la TRC tradicional (estimulación biventricular) cuando es aplicada en pacientes con una activación preservada de la rama izquierda, resulta en prolongación del tiempo de activación del ventrículo izquierdo relativo al ritmo sinusal ${ }^{13}$.

Sharma, et al. ${ }^{14}$, en un estudio realizado en 2018 , evaluaron el impacto de la estimulación hisiana permanente en un grupo de pacientes con BRDHH e indicación para resincronización cardiaca por la duración del QRS y tras 15 meses de seguimiento demostraron una reducción significativa de la duración del QRS (158-127 ms), incremento de la FEVI (31-39\%), mejoría en la clase funcional.

Se ha documentado previamente en múltiples informes en la literatura, y en este caso se demostró, que la técnica de estimulación hisiana es útil, segura y eficaz en pacientes con insuficiencia cardiaca, BRDHH y QRS 


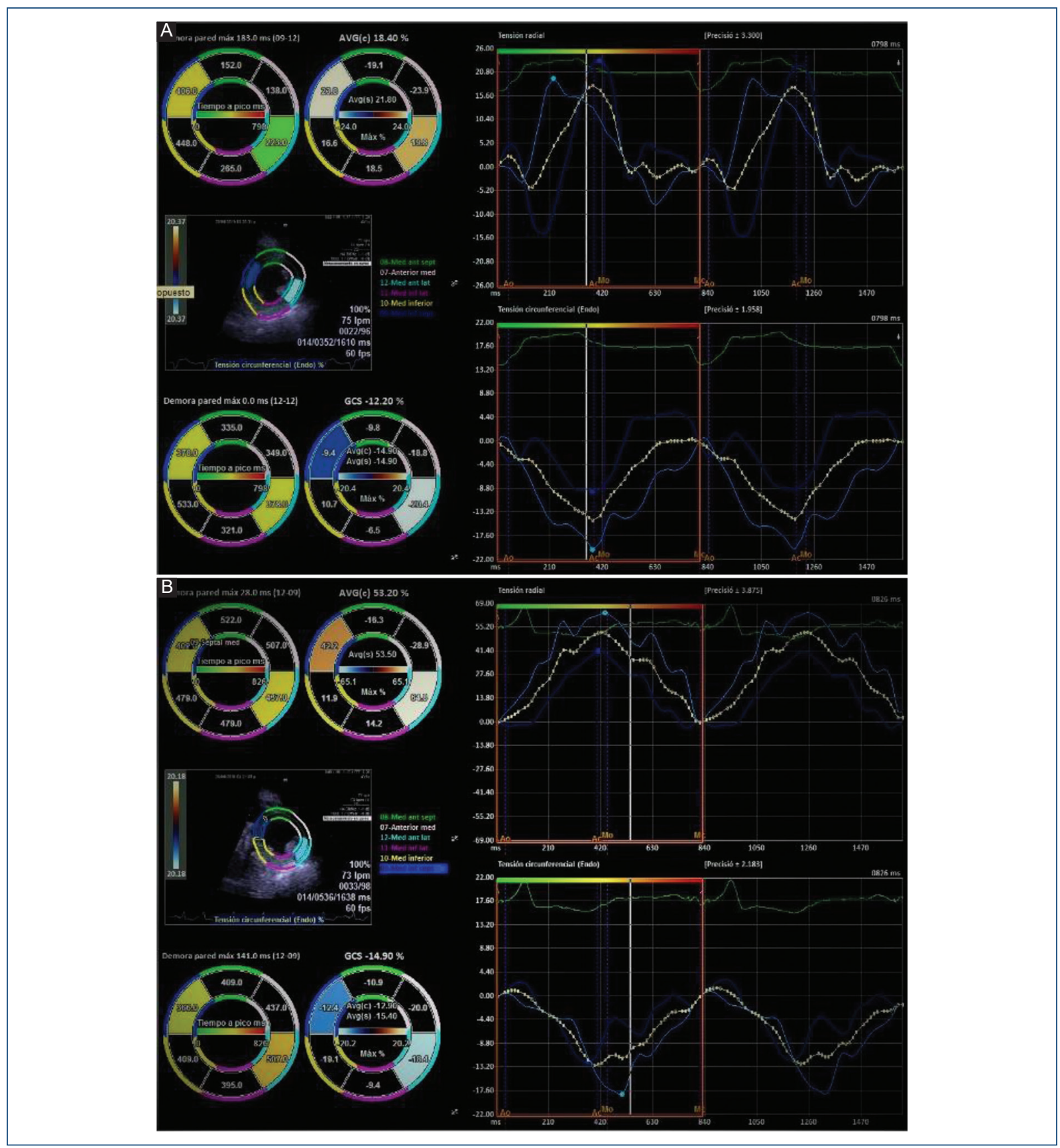

Figura 3. Deformación radial y circunferencial mediante técnica de rastreo de marcas (speckle tracking) con análisis por vectores. A: antes de la estimulación hisiana se muestra asincronía intraventricular con un tiempo de retraso en el pico de la deformación radial de 183 ms entre la pared anteroseptal e inferior, con un índice de retraso de deformación del $32 \%$. B: posterior a la estimulación se muestra reducción del tiempo de retraso en el pico de deformación radial de hasta $28 \mathrm{~ms}$ y con mejoría también del índice de retraso de la deformación anteroseptal.

ancho como terapia alternativa a la resincronización cardiaca convencional, con buenos resultados a corto-mediano plazo en pacientes adecuadamente seleccionados con duración del QRS > 150 ms en el ECG basal, o en caso de ser menor, la disincronía intraventricular debe evaluarse mediante ecocardiograma transtorácico idealmente con strain radial.
En nuestro caso, dada la mejoría que observamos de la FEVI del 17 al $36.6 \%$ cabe la posibilidad de que este grupo de pacientes pudieran salir de la indicación de implante de desfibrilador como prevención primaria, para lo cual será necesario observar series de estudios multicéntricos en los cuales se pueda evaluar la respuesta. 


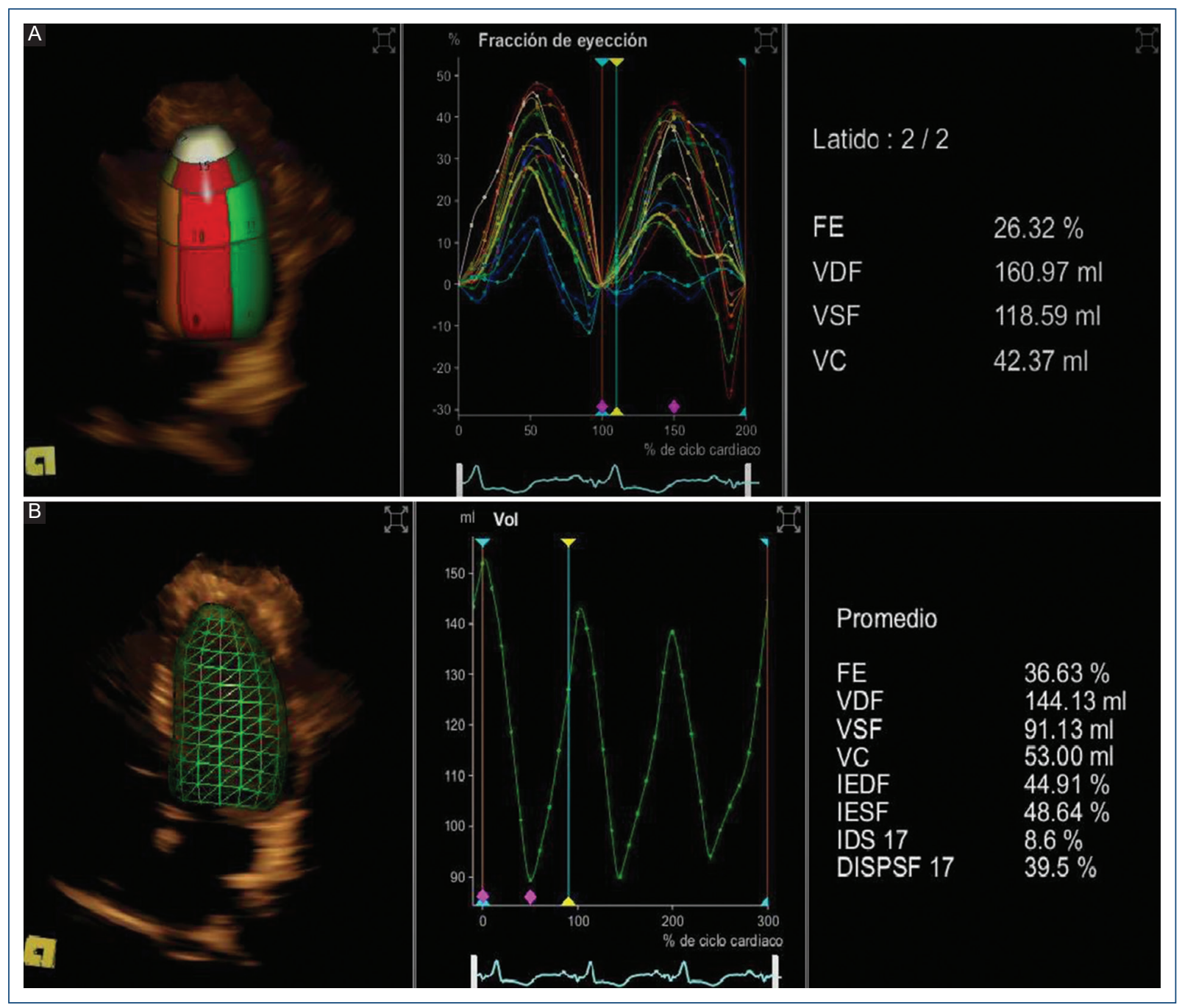

Figura 4. Cuantificación de la fracción de eyección ventricular izquierda (FEVI) 3D. A: la curva fracción de expulsión (FE)/tiempo es del 26.32\%. B: posterior a la terapia de resincronización cardiaca y estimulación hisiana es del $36.6 \%$ se observa una mejoría del 10.31 \% de la FEVI. El volumen diastólico final (VDF) disminuyó de $160.97 \mathrm{~mL}$ (A) a $144.13 \mathrm{~mL}$ (B). El volumen sistólico final (VSF) disminuyó de $118.59 \mathrm{~mL}$ (A) a $91.13 \mathrm{~mL}$ (B). El volumen latido (VC) aumentó de $42.37 \mathrm{~mL}$ (A) a $53 \mathrm{~mL}$ (B). En el gráfico B, curva volumen/tiempo, se identificó mejoría en $10.63 \mathrm{~mL}$ en el volumen latido con respecto a la basal.

\section{Financiamiento}

Ninguno.

\section{Conflicto de intereses}

Los autores declaran no tener conflicto de intereses.

\section{Responsabilidades éticas}

Protección de personas y animales. Los autores declaran que los procedimientos seguidos se conformaron a las normas éticas del comité de experimentación humana responsable y de acuerdo con la
Asociación Médica Mundial y la Declaración de Helsinki.

Confidencialidad de los datos. Los autores declaran que han seguido los protocolos de su centro de trabajo sobre la publicación de datos de pacientes.

Derecho a la privacidad y consentimiento informado. Los autores han obtenido el consentimiento informado de los pacientes y/o sujetos referidos en el artículo. Este documento obra en poder del autor de correspondencia.

\section{Bibliografía}

1. Curtis AB, Worley SJ, Adamson PB, Chung E, Niazi I, Sherfesee L, et al. Biventricular pacing for atrioventricular block and systolic dysfunction. $\mathrm{N}$ Engl J Med. 2013;368:1585-93. 
2. Brignole M, Auricchio A, Baron-Esquivias G, Bordachar P, Boriani G, Breithardt OA, et al. 2013 Guidelines on cardiac pacing and cardiac resynchronization therapy. Eur Heart J. 2013;34:2281-29.

3. Tang AS, Wells GA, Talajic M, Arnold MO, Sheldon R, Connolly S, et al. Resynchronization-Desfibrillation for Ambulatory Heart Failure Trial Investigators. Cardiac-resynchronization therapy for mild-to-moderate heart failure. N Engl J Med 2010;363:2385-95.

4. Epstein AE, DiMarco JP, Ellenbogen KA, Estes NA III, Freedman RA, Gettes LS, et al. 2012 ACCF/AHA/HRS focused update incorporated into the ACCF/AHA/HRS 2008 guidelines for device-based therapy of cardiac rhythm abnormalities: a report of the American College of Cardiology Foundation/American Heart Association Task Force on Practice Guidelines; Heart Rhythm Society. J Am Coll Cardiol. 2013;61: e6-e75.

5. Sharma PS, Dandamudi G, Naperkowski A, Oren JW, Storm RH, Ellenbogen KA, et al. Permanent His-bundle pacing is feasible, safe, and superior to right ventricular pacing in routine clinical practice. Heart Rhythm. 2015;12:305-12.

6. Vijayaraman P, Herweg B, Ellenbogen K, Gajek J. His-optimized cardiac resynchronization therapy to maximize electrical resynchronization. Circ Arrhythm Electrophysiol. 2019:12:e006934.

7. Dandamudi G, Vijayaraman P. How to perform permanent His Bundle pacing in routine clinical practice. Heart Rhythm. 2016;13:1362-66.
8. Verhaert D, Grimm RA, Puntawangkoon C, Wolski K, De S, Wilkoff BL, et al. Long-term reverse remodeling with cardiac resynchronization therapy. Circulation. 2010;122:985-92.

9. Peschar M, de Swart H, Michels KJ, Reneman RS, Prinzen FW. Left ventricular septal and apex pacing for optimal pump function in canine hearts. J Am Coll Cardiol. 2003;41:1218-26.

10. Chandra R, Zolty R, Palma E. A left hemiblock improves cardiac resynchronization therapy outcomes in patients with a right bundle branck block. Clin Cardiol. 2010;33:89-93.

11. Varma N. Left ventricular conduction delays and relation to QRS configuration in patients with left ventricular dysfunction Am J Cardiol. 2009;103:1578-85.

12. Sing JP, Berger RD, Doshi RN, Lloyd m, Moore D, Stone J, et al.; ENHANCE CRT Study Group. Targeted left ventricular lead implantation in non-left bundle branch block patients: primary results of the ENHANCE CRT study. JACC Clin Electrophysiol. 2020;6(9):1171-81.

13. Ploux S, Whinnett Z, Lumens J, Denis A, Zemmoura A, De Guillebon M, et al. Acute hemodynamic response to biventricular pacing in the heart failure patients with narrow, moderately, and severly prolonged QRS duration. Heart Rhythm. 2012;9:1247-50.

14. Sharma PS, Naperkowski A, Bauch TD, Chan JY, Arnold AD, Whinnett ZI, et al. Permanent His bundle pacing for cardiac resynchronization therapy in patients with heart failure and right bundle branch block. Circ Arrhythm Electrophysiol. 2018;11:e006613. 